\title{
Mining Frequent Patterns in Large Scale Databases Using Adaptive FP-Growth Approach
}

\author{
Doo Hee Han and Zhang Nv
}

\begin{abstract}
Frequent Patterns (FPs) are extremely vital in knowledge discovery and data mining process, for instance, mining of association rules, correlations etc. Several existing incremental mining schemes are mostly Apriori-based, which are not easily adaptable to solve association rule mining. FPtree is a compact representation of transaction database that includes frequency information of the entire relevant FPs in a dataset. Mining association rules in the midst of items in a large database is one of the most vital data mining problems. An earlier scheme proposed a model that is able to mine in transactional database, however that approach is not able to manage the problem of changing the memory dynamically. Hence, in order to solve this complication, here a hybrid of two algorithms is proposed that has the potential of handling the dynamic change of memory, dynamic databases and also to solve the complication of association rule mining problems. Hence, memory can be effectively utilized in large scale transaction database.
\end{abstract}

Keywords--- Frequent Patterns, Transaction Database, Apriori Algorithm, Association Rule, FP Tree.

\section{INTRODUCTION}

$\mathrm{D}$ ATA mining is a term that refers to searching a large data set in an attempt to detect hidden or low-level patterns. Data mining is becoming increasingly common in both the private and public sectors. Industries such as banking, insurance, medicine, and retailing commonly use data mining to reduce costs, enhance research, and increase sales. In the public sector, data mining applications initially were used as a means to detect fraud and waste, but have grown to also be used for purposes such as measuring and improving program performance. However, some of the homeland security data mining applications represent a significant expansion in the quantity and scope of data to be analyzed.

In this paper association rule mining defines finding frequent patterns, associations, correlations, or causal structures among sets of items or objects in transaction databases, relational databases, and other information repositories. The main Applications are Basket data analysis, cross-marketing, catalog design, loss-leader analysis, clustering, classification, etc.[1]

Association rule mining is to find out association rules that satisfy the predefined minimum support and confidence from a given database. The problem is usually decomposed into two subproblems. One is to find those itemsets whose occurrences

Doo Hee Han, Tsinghua University, China.

Zhang Nv, Nanyang Technological University of Singapore (NTU). DOI : 10.9756/BIJIEMS.8326 exceed a predefined threshold in the database; those itemsets are called frequent or large itemsets. The second problem is to generate association rules from those large itemsets with the constraints of minimal confidence.

The task of discovering all frequent itemsets is quite challenging. The search space is exponential in the number of items occurring in the database. The support threshold limits the output to a hopefully reasonable subspace. Also, such databases could be massive, containing millions of transactions, making support counting a tough problem. Frequent pattern mining has been studied for over a decade with tons of algorithms developed

- Apriori

- FPgrowth (it only considered in this paper)

Large data are data sets characterized by a large number of columns (i.e., attributes) and few rows (i.e., transactions). We propose in this paper a method to mine frequent from a large data without transposing the data set. The key idea is to use extension of a pattern to check these constraints, because the extension has few objects in large databases.[3]We show a new property to compute the extension of a pattern and a new pruning criterion. Their simultaneous use is on the core of the hybrid of adaptive and bounded algorithm that we propose to extract the frequent patterns from large database.

\section{RELATED WORK}

Han et al. proposed FP-growth algorithm [4] to discover frequent patterns from FP-tree. FP-growth traverses the FPtree in a depth-first manner. It requires only two scans of the dataset to construct FP-tree, unlike Apriori algorithm [6] that makes multiple scans over the dataset. Since the introduction of FP-growth algorithm three major algorithms have been proposed, namely AFPIM, CATS tree, and CanTree that have adopted FP-tree for incremental mining of frequent patterns. Several efficient algorithms have been proposed for finding frequent itemsets and the association rules are derived from the frequent itemsets, such as the Apriori[4] and DHP algorithms.

Apriori: The Apriori heuristic achieves good performance gained by (possibly significantly) reducing the size of candidate sets. However, in situations with a large number of frequent patterns, long patterns, or quite low minimum support thresholds, an Apriori-like algorithm may suffer from the following two nontrivial costs:

- It is costly to handle a huge number of candidate sets.

- It is tedious to repeatedly scan the database and check a large set of candidates by pattern matching, which is especially true for mining long patterns. 


\section{A. Cats Tree}

CATS tree (Compressed and Arranged Transaction Sequence Tree) [5] mainly for interactive mining. The CATS tree extends the idea of the FP-tree to improve storage compression, and allows frequent-pattern mining without the generation of candidate itemsets. Once cats is built, it can be used for multiple frequent pattern mining with different supports. The idea of tree construction is as follows. It requires one database scan to build the tree. New transactions are added at the root level. At each level, items of the new transaction are compared with children (or descendant) nodes. If the same items exist in both the new transaction and the children (or descendant) nodes, the transaction is merged with the node at the highest frequency level. The remainder of the transaction is then added to the merged nodes, and this process is repeated recursively until all common items are found. Any remaining items of the transaction are added as a new branch to the last merged node. If the frequency of a node becomes higher than its ancestors, then it has to swap with the ancestors so as to ensure that its frequency is lower than or equal to the frequencies of its ancestors. Let us consider the following example to gain a better understanding of how the CATS tree is constructed.

Example 1: Consider the following database

\begin{tabular}{|l|l|}
\hline Tid & Transactions \\
\hline 1 & $\mathrm{r}, \mathrm{s}, \mathrm{t}, \mathrm{l}$ \\
\hline 2 & $\mathrm{q}, \mathrm{s}, \mathrm{t}$ \\
\hline 3 & $\mathrm{p}, \mathrm{q}, \mathrm{r}, \mathrm{t}$ \\
\hline 4 & $\mathrm{p}, \mathrm{s}, \mathrm{t}, \mathrm{u}$ \\
\hline 5 & $\mathrm{p}, \mathrm{r}, \mathrm{s}, \mathrm{t}$ \\
\hline
\end{tabular}

\begin{tabular}{|l|l||}
\hline Tid & Transactions \\
\hline 1 & $\mathrm{t}, \mathrm{s}, \mathrm{r}$ \\
\hline 2 & $\mathrm{t}, \mathrm{s}$ \\
\hline 3 & $\mathrm{t}, \mathrm{r}, \mathrm{p}$ \\
\hline 4 & $\mathrm{t}, \mathrm{s}, \mathrm{p}$ \\
\hline 5 & $\mathrm{t}, \mathrm{s}, \mathrm{r}, \mathrm{p}$ \\
\hline
\end{tabular}

Figure 1: a) Initial Dataset b) Projected Dataset with Minthreshold $=50 \%$

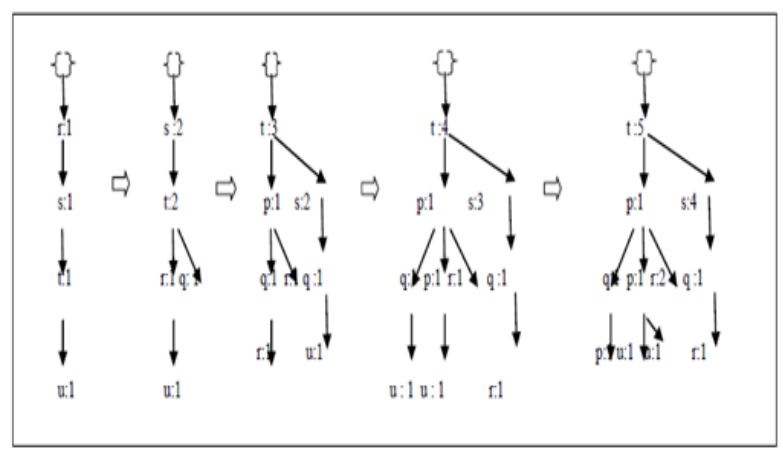

Figure 2: Stepwise Construction of CATS Tree While Processing Each Transaction

Figure 2: shows how CATS tree is constructed considering the dataset of Figure 1.However, CATS tree too has two limitations. First, for each new transaction it is required to find the right path for the new transaction to merge in. Second, it is required to swap and merge the nodes during the updates, as the nodes in CATS tree are locally sorted.

\section{B. Can Tree}

CanTree (Canonical-order Tree), that captures the content of the transaction database and orders tree nodes according to some canonical order.[7] The canonical order can be determined by the user prior to mining process. Canonical ordering can be lexicographic or based on certain property values of items By exploiting its nice properties, the CanTree can be easily maintained when database transactions are inserted, deleted, and/or modified. For example, the CanTree does not require adjustment, merging, and/or splitting of tree nodes during maintenance. No rescan of the entire updated database or reconstruction of a new tree is needed for incremental updating.

\section{FP Tree}

In this paper, we consider the FP-growth approach, Han et al. [4] suggested that the FP-tree can be saved in the secondary memory if it cannot be accommodated in main memory. FP tree is a fundamental data structure for FP growth. Their proposal is a disc-resident version of the FP-tree that would use the $B+$-tree, which is a very popular structure. They proposed that the top level nodes of the $B+$-tree can be split based on the roots of item prefix subtrees, and the second level based on the common prefix paths, and so on. They also proposed a group accessing scheme to improve the I/O access related to the disc-based nodes so that one would traverse nodes that are in memory first before fetching other nodes from disc.

The general idea of mining frequent pattern using FP tree is divide and conquer method, it happens recursively grow frequent pattern path. The method of mining the frequent pattern is for each item, it construct its conditional pattern base, then its conditional FP tree.Again, repeat the process until the resulting FP tree is empty, or it contains only one path. For this we considered an algorithm as Adapative and Bounded memory based approach to mine frequent pattern.

\section{FP TREE CONSTRUCTION}

We mainly present a running example to demonstrate the different aspects related to Han et al.'s [2] FP-growth and FPtree as the underlying structure because both are required to thoroughly understand the approach proposed in this paper. In this section, we describe the construction procedure of the FPtree and its algorithm via an illustrative example.

Lets consider an example for mine frequent pattern, Table I shows a transactional database with six transactions. Every row is identified by TID and consists of a set of items that occur together in a single transaction. In order to construct the FP-tree from this transactional database, first scan the database once to collect the count of the items present in the database. Then, sort the items according to their frequencies in descending order to build the frequent item list. Only items that meet the minimum support threshold are considered for building the FP-tree. All items with their frequency counts are shown are the items sorted according to their frequencies in descending order. 
Table I: A Transactional Database as Running Example

\begin{tabular}{ccc}
\hline TID & liems bought & (Ondered) frequent items \\
\hline 100 & $f, a, c, d, g, i, m, p$ & $f, c, a, m, p$ \\
200 & $a, b, c, f, l, m, o$ & $f, c, a, b, m$ \\
300 & $b, f, h, j, o$ & $f, b$ \\
400 & $b, c, k, s, p$ & $c, b, p$ \\
500 & $a, f, c, e, l, p, m, n$ & $f, c, a, m, p$ \\
\hline
\end{tabular}

Only items that meet the minimum support threshold are shown in tabulation. Finds and arranged the frequent pattern with table (1). Once the tree is constructed; it is possible to mine for frequent patterns using the FP-Growth approach, which is a recursive mining approach that divides the mining task into separate smaller ones. The FP-Growth approach starts mining by looking up the header table and selecting items that meet the specified minimum support criteria.

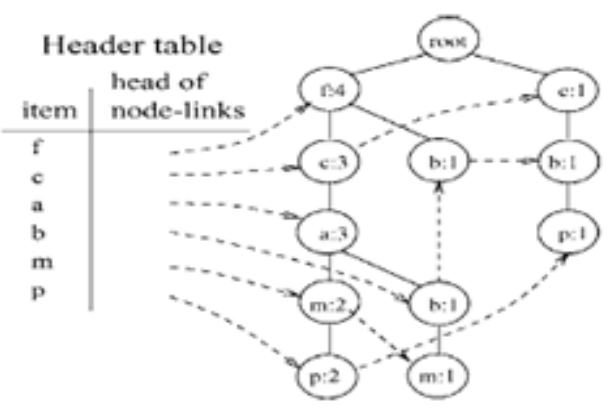

The FP-troe in Example 1.

After construction of FP tree, child node is added to the branch while maintaining the prefix path property of the FPTree. But the time when this prefix path is created and where in the linear memory space the nodes of the prefix path are placed, depend on the order by which the transactions are read and added to the file. So nodes are numbered and store in linear memory as array like fashion. Basically FP growth suffers from spatial and temporal localities. To improve the spatial and temporal localities from FP growth are:

- Approximate Sorting

- Reorganizing Data, I/O Conscious FP-Tree

- Reorganizing Computation, Page Blocking

- Issues Associated With Relying on VMM

\section{PROBLEM DEFINITION}

In this section, we will describe the problem in this paper. Let's consider raw transactional database, total main memory (M), secondary storage(S) is available. Based on this, finds all frequent patterns which corresponds to database and its support threshold. Similarly, an ARM model, finds from database, all association rules that meet the desired thresholds for support $\sigma$ and confidence $c$. traditionally, there are no restrictions on the use of $M$ and $S$. A particular mining model may use any portion of $M$ and/or $S$ that the model requires to find the frequent patterns/association rules. Moreover, the model must assume that its memory is limited and hence cannot grow beyond the already specified size; it is possible that other parts of the main memory may be assigned to other tasks that are independent of the mining task.

\section{BOUNDED AND ADAPTIVE APPROACH}

In this section, we present the architecture of the proposed frequent pattern mining model, which can mine frequent patterns management unit of the model allocates a contiguous portion of main memory $M . B$, where structures required for the mining task, which includes the following:

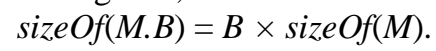

This allocated contiguous block of memory stores all the data using a bounded portion of the main memory. Each component of the proposed architecture shown in Figure. Based on this ratio $B$, the memory 1) FP-nodeslist(which is an array of FP-nodes where the initial FP-tree, and subsequently, the conditional FP-trees are realized);

1. Memory Tree Location Table (MTLT);

2. Disc Tree Location

3. Table (DTLT);

4. Item Location Table (ILT);

5. Count Location Table (CLT); and

6. FB to load blocks of the disc-based prefix tree

\section{A. Secondary Storage}

Our proposed mining model realizes the secondary storage $S$ as a collection of file-based structures. If at any certain point of the mining process, the MMU cannot accommodate the data structures in $M . B$, it translates the overflowed data structures into linear file-based structures.

\section{B. Memory Management Unit}

The MMU lies all the translations necessary to save the overflowed memory-based data structures (like FP-trees, MTLT, DTLT, etc.) into the secondary storage $S$ as file-based structures. It also loads the data structures from the file-based structures to the desired memory-based structures when required. The tree translation unit translates a memory-based FP-tree which is located at the FP-nodes-list into a disc (file)based version of I/O-conscious prefix-tree. Next, the tree is divided into two blocks, where each block size is less than the size of FB. Next, each block is saved into a file structure as an I/O-conscious prefix-tree.

Disc Tree Location Table (DTLT) is an R-tree- [8] based structure that stores the knowledge of Prefix-Treediscnode-Id ranges associated with each prefix-tree Moreover, it makes sure that the DTLT is always maintained using only a bounded portion of M.B. It also keeps a hash table associated with the DTLT structure to easily identify nodes of the DTLT structure that are actually in memory and those that are in a disc-based file. Actually, if a node is not present in the hash table, this subunit assumes the node is stored on disc and subsequently loads the node into memory and creates a corresponding entry in the hash table. 

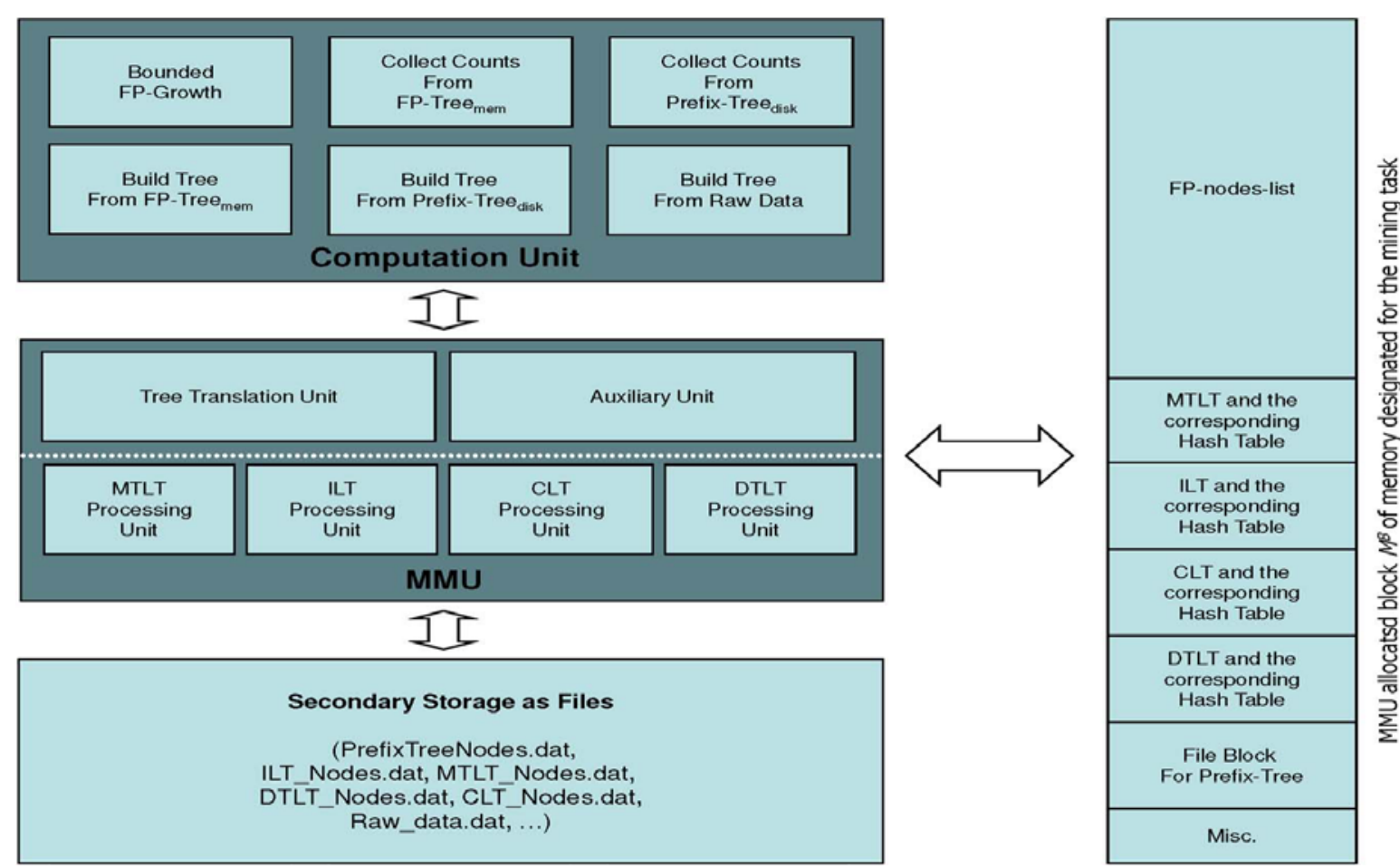

\section{Secondary Storage as Files \\ (PrefixTreeNodes.dat, \\ ILT_Nodes.dat, MTLT Nodes.dat, \\ DTLT_Nodes.dat, CLT_Nodes.dat, Raw_data.dat, ...)}

\section{FIG -Architecture of Bounded and Adaptive}

The ILT structure is also an R-tree based structure, which stores the node-link pointers of the prefix-trees. Each ILT internal node contains dimensionality information as treeIdRange, itemIdRange, and nodeIdRange; whereas, a leaf node holds the nodeIds of a particular item in a particular prefixtree block, in addition to treeId, itemId, and nodeIdRange. The ILT processing unit does all the manipulation of the ILT structure in a way similar to how the DTLT processing unit processed.

MTLT is an R-tree-based structure which keeps track of the FP-trees that are currently present in memory. MTLT structure uses this id as dimensionality information. The leaf node of the MTLT structure contains a pointer to the root node of the particular FP-tree in memory and also a file pointer to the header table which if not used is saved on the disc.

The CLT structure stores the count/frquency list of all the items in a particular prefixtree. Auxiliary unit is the subunit manipulates the data structures that are placed on the Misc and the (File Block) FB portion of M.B; $\mathrm{M}$ is the total main memory and B is the bounded portion of primary memory .It is mainly used by some of the subunits of the MMU and the computation unit.

\section{CONCLUSION AND FUTURE WORK}

In this work, an approach of frequent pattern mining based on the adaptive FP-Growth approach is presented by handling the memory allocation dynamically. Also the proposed approach have been compared with the present work in case of the performance metrics such as the time taken and the memory usage by the approaches for the frequent pattern mining. From the results it was found that the proposed work scale up the performance of the approach of frequent pattern mining from the large database and also able to have a dynamic memory management by developing a specialized memory management unit and by eradicating the concept of behaviour of depending up on the virtual Memory Management. This method can be further used for research studies in order to make the approach more specific and to be handled for any range of the large database handling the different types of the data.

\section{REFERENCES}

[1] M. Adnan and R. Alhajj, "A Bounded and Adaptive Memory-Based Approach to Mine Frequent Patterns From Very Large Databases", IEEE Transactions on Systems, Man, and Cybernetics-Part B: Cybernetics, Vol. 41, No. 1, 2011.

[2] S. Kotsiantis and D. Kanellopoulos, “Association Rules Mining: A Recent Overview", GESTS International Transactions on Computer Science and Engineering, Vol. 32, No.1, Pp. 71-82, 2006.

[3] B. Goethals," Survey on Frequent Pattern Mining”, Univ. of Helsinki, 2003.

[4] R. Agrawal, T. Imielinski and A.N. Swami, "Mining association rules between sets of items in large databases", ACM SIGMOD'93, Pp. 207-216, 1993.

[5] C. H'ebert and B. Cr'emilleux, "Mining Frequent $\delta$-Free Patterns in Large Databases", International Conference on Discovery Science , Springer-Verlag Berlin Heidelberg 2005.

[6] R.J. Bayardo, "Efficiently mining long patterns from databases", Proc. SIGMOD, Pp. 85-93, 1998.

[7] J. Han, J. Pei, Y. Yin and R. Mao, "Mining Frequent Patterns without Candidate Generation: A Frequent-Pattern Tree Approach”, Data Mining and Knowledge Discovery, Vol. 8, No. 1, Pp.53-87, 2004.

[8] W. Cheung and O.R. Za" 1ane, "Incremental mining of frequent patterns without candidate generation or support constraint”, Proc. IDEAS, Pp. 111-116, 2003.

[9] R. Agrawal, T. Imielinski and A. Swami, "Mining association rules between sets of items in large databases", Proc. of ACM-SIGMOD, Pp. 207-216, 1993.

[10] C.S. Leung, Q.I. Khan and T. Hoque, "CanTree: a tree structure for efficient incremental mining of frequent patterns", IEEE International Conference on Data Mining, Fifth, 2005.

[11] A. Guttman, "R-trees: A dynamic index structure for spatial searching", Proc. ACM SIGMOD, Pp. 47-57, 1984. 